\title{
Line bundles on complex tori and a conjecture of Kodaira
}

\author{
Jean-Pierre Demailly, Thomas Eckl and Thomas Peternell
}

Mathematics Subject Classification (2000). 32Q15, 32J27.

Keywords. Kähler manifold; deformation; vector bundles.

\section{A conjecture of Kodaira}

A fundamental question in Kähler geometry asks whether any compact Kähler manifold can be realised as a deformation of a projective manifold. This is made more precise in the following

Definition 1. A compact Kähler manifold $X$ is said to be algebraically approximable, or almost algebraic, if there exists a complex manifold $\mathcal{X}$ and a surjective holomorphic submersion $\pi: \mathcal{X} \rightarrow \Delta$ to the unit disc $\Delta \subset \mathbb{C}$ such that the fibers $\mathcal{X}_{t}=\pi^{-1}(t)$ satisfy $\mathcal{X}_{0} \simeq X$ and there is a sequence $\left(t_{k}\right)$ converging to 0 such that all $X_{t_{k}}$ are projective.

In [Kod63] Kodaira proved that every Kähler surface is almost algebraic, and it was a standard conjecture, known as the Kodaira conjecture, that this should be also true in higher dimensions. In particular, according to that conjecture, every rigid compact Kähler manifold should have been algebraic.

However recently, a few months after this paper was completed, C.Voisin [Vo04a] came up with a counterexample: she constructed a rigid non-algebraic Kähler threefold, arising as a blow-up of a complex torus. Later Oguiso [Og04] constructed a simply connected counterexample. During the final revision of this paper, C. Voisin [Vo04b] even announced the construction of Kähler manifolds such that no smooth bimeromorphic model can be deformed to a projective complex manifold, thereby showing that a weakened "bimeromorphic version" of the Kodaira conjecture does not hold either.

However, even with the original version of the Kodaira conjecture, we still believe that there are important classes of compact Kähler manifolds for which algebraic approximation is possible. Such a class might be the class of minimal compact Kähler manifolds, i.e. manifolds with $K_{X}$ nef, since blow-up tricks used to manipulate tori are 
then forbidden. Another such class should be the class of compact Kähler manifolds with hermitian seminegative canonical bundle (or, even more generally, with $-K_{X}$ nef). A structure theorem for compact Kähler manifolds of this type states that they have a finite étale covering mapping surjectively onto the Albanese torus, and the fibers of the Albanese map are products of Calabi-Yau manifolds, hyperkähler manifolds or manifolds $X$ with $-K_{X}$ semipositive and $H^{0}\left(X, \Omega_{X}^{\otimes m}\right)=0$ for all $m>0$ [DPS96]. The latter manifolds are projective algebraic, while tori and hyperkähler manifolds are algebraically approximable, so there is indeed a very good hope to reach a proof for this class; the very special case of numerically flat projective bundles over complex tori follows in fact from Proposition 2 below.

The main idea is the following easy general argument for projective bundles over tori, which asserts that the projective bundle structure survives by deformation.

Proposition 2. Let $X$ be a compact Kähler manifold which has a $\mathbb{P}_{r}$-bundle structure $X \rightarrow A$ over some complex torus A. Then for every deformation $X \rightarrow S$ with $X_{0} \simeq X$, the nearby fibers $\mathcal{X}_{t}$ have a $\mathbb{P}_{r}$-bundle structure $\mathcal{X}_{t} \rightarrow \mathcal{A}_{t}$ where $\mathcal{A}$ is a deformation of $A$ in a neighborhood of $t=0$. Moreover, if $X=\mathbb{P}(V)$ for some vector bundle $V$ on $A$, then $\mathcal{X}_{t}=\mathbb{P}\left(V_{t}\right)$ for a suitable deformation $V_{t} \rightarrow \mathcal{A}_{t}$ of $V \rightarrow A$.

Proof. We look at the relative Albanese map $\alpha: \mathcal{X} \rightarrow \mathcal{A}$. Then $\mathcal{A} \rightarrow S$ is a deformation of tori such that $\alpha_{t}: \mathcal{X}_{t} \rightarrow \mathcal{A}_{t}$ is the Albanese map for each $t \in S$. Since $\alpha_{0}$ is a submersion, $\alpha_{t}$ should be also a submersion $t$ in a neighborhood $U \subset S$ of 0 , and the fibers of $\alpha_{t}$ are deformations of $\mathbb{P}_{r}$. Since $\mathbb{P}_{r}$ is undeformable, we conclude that $\alpha_{t}: \mathcal{X}_{t} \rightarrow \mathcal{A}_{t}$ is also a $\mathbb{P}_{r}$-bundle for small $t$. Now, the fact that $\chi_{t}=\mathbb{P}\left(V_{t}\right)$ is equivalent to the fact that the relative anticanonical bundle $K_{\mathcal{X}_{t} / \mathcal{A}_{t}}^{1}$ has an $(r+1)$-root $L_{t}$ on $\mathcal{X}_{t}$, in which case $V_{t}=\left(\alpha_{t}\right)_{*}\left(L_{t}\right)$. However, the obstruction for a line bundle to have an $(r+1)$-root lies in $H^{2}\left(\mathcal{X}_{t}, \mathbb{Z} /(r+1) \mathbb{Z}\right)$. This is a discrete locally constant coefficient system, so if the obstruction vanishes for $t=0$, it must also vanish on the connected component of 0 in $U \subset S$.

Proposition 2 more generally holds for arbitrary projective bundles over compact manifolds and even for bundles whose fibers are rigid manifolds without holomorphic 1 -forms; the proof is slightly more involved and is given in the last section.

In view of this, it is natural to look at the following potential candidate for a counter-example: Start with a 3-dimensional complex torus $A$ with Picard number $\rho(A) \geq 3$. Let $L_{i} \in \mathrm{NS}(A)$ be (numerical equivalence classes of) linearly independent holomorphic line bundles over $A$. Let $U \subset \mathbb{C}^{9}$ be a neighborhood of $[A]$ in the universal deformation space of $A$. As explained in the next section, every $L_{i}$ determines a 3-codimensional subspace $V_{i}=V\left(L_{i}\right)$ in $U$ such that $c_{1}\left(L_{i}\right)$ is $(1,1)$, i.e. $L_{i}$ is a holomorphic line bundle on $A^{\prime}$ if and only if $\left[A^{\prime}\right] \in V_{i}$. 
Vol. 80 (2005)

Now we make the following Assumption:

The intersection of the $V_{i}$ 's has the expected dimension 0 , i.e.

(*) $\quad V_{1} \cap V_{2} \cap V_{3}$ contains $\{A\}$ as an isolated point.

Then consider the 6-dimensional manifold

$$
Y=\mathbb{P}\left(\mathcal{O}_{A} \oplus L_{1}\right) \times_{A} \mathbb{P}\left(\mathcal{O}_{A} \oplus L_{2}\right) \times_{A} \mathbb{P}\left(\mathcal{O}_{A} \oplus L_{3}\right) .
$$

This is a $\mathbb{P}_{1}^{3}$-bundle over $A$ with projection $\pi: Y \rightarrow A$. In each subspace $\mathbb{P}\left(\mathcal{O}_{A} \oplus L_{i}\right)$ there is a section $Z_{i}$ at infinity given by the direct summand $\mathcal{O}_{A}$. This gives a section $Z$ of $\pi$ by selecting over every $a \in A$ the point $\left(x_{1}, x_{2}, x_{3}\right)$, where $\left\{x_{i}\right\}=Z_{i} \cap \pi^{-1}(a)$.

Proposition 3. The blow up $\sigma: X \rightarrow Y$ of $Z \subset Y$ is rigid in the sense that there is no positive-dimensional family of deformations of $X$.

Proof. Notice that, denoting by $\mathbb{P}_{1}^{3}(x)$ the blow up of $\mathbb{P}_{1}^{3}$ in one point, $X$ is a $\mathbb{P}_{1}^{3}(x)$-bundle over $A$. So let $\left(X_{t}\right)$ be a deformation of $X=X_{0}$ over the 1-dimensional unit disc $\Delta$. The first step is to proof that, possibly after shrinking $\Delta$, every $X_{t}$ is a $\mathbb{P}_{1}^{3}(x)$-bundle over its (3-dimensional) Albanese torus $A_{t}$. In fact, $q\left(X_{t}\right)=3$ for all $t$ and the Albanese map $\alpha_{t}$ is smooth for small $t$. In order to prove that $\alpha_{t}$ is a $\mathbb{P}_{1}^{3}(x)$-bundle, it suffices to show that $\mathbb{P}_{1}^{3}(x)$ is rigid, i.e. every small deformation of $\mathbb{P}_{1}^{3}(x)$ is again $\mathbb{P}_{1}^{3}(x)$.

In fact, let $Z=\mathbb{P}_{1}^{3}(x)$ for simplicity of notations. Let $\tau: Z \rightarrow \mathbb{P}_{1}^{3}$ be the blow-up map with exceptional divisor $E \simeq \mathbb{P}_{2}$. Then there is an exact sequence

$$
0 \rightarrow T_{Z} \rightarrow \tau^{*} T_{\mathbb{P}_{1}^{3}} \rightarrow T_{E}(-1) \rightarrow 0 .
$$

Since $\operatorname{dim} H^{0}\left(T_{\mathbb{P}_{1}^{3}}\right)=9, \operatorname{dim} H^{0}\left(T_{Z}\right)=6, H^{0}\left(T_{E}(-1)\right)=3$ and $H^{1}\left(\tau^{*} T_{\mathbb{P}_{1}^{3}}\right)=0$, by taking cohomology of the above exact sequence it follows

$$
H^{1}\left(T_{Z}\right)=0,
$$

in particular $Z$ is rigid.

Let $\mathcal{X}$ be the total space of $\left(X_{t}\right)$ and let $\pi: \mathcal{X} \rightarrow \mathcal{A}$ be the relative Albanese map for $X \rightarrow \Delta$. Then $\mathcal{A} \rightarrow \Delta$ is a torus bundle; let $A_{t}$ be the fiber over $t$, so that $A=A_{0}$. Now the exceptional divisor $D$ of $\sigma$ moves in $X$. This is easy to see by considering $D \cap \pi^{-1}(a)=\mathbb{P}_{2}$ for $a \in A$. In fact, the normal bundle of this $\mathbb{P}_{2}$ is $\mathcal{O}(-1) \oplus \mathcal{O}^{4}$, so that the $\mathbb{P}_{2}$ moves and forces $D$ to move. Therefore one obtains a fiberwise blow-down $X \rightarrow \mathcal{Y}$ inducing the birational map $\sigma: X \rightarrow Y$. Of course there is a factorisation $\mathcal{X} \rightarrow \mathcal{Y} \rightarrow \mathcal{A}$ and $\mathcal{y} \rightarrow \mathcal{A}$ is a $\mathbb{P}_{1}^{3}$-bundle. Again let $Y_{t}$ be the fiber over $t$. Next it is shown that it is possible to write

$$
Y_{t}=Y_{1, t} \times_{A_{t}} Y_{2, t} \times_{A_{t}} Y_{3, t}
$$


with $\mathbb{P}_{1}$-bundles $Y_{i, t} / A_{t}$, and this can be done simultaneously, i.e. the $\left(Y_{i, t}\right)$ form a family $y_{i}$. The most economic way to do that is to note that the relative Picard number $\rho(\mathcal{H} / \mathcal{A})$ equals 3 since $\rho\left(Y_{0} / A_{0}\right)=3$ and since $K_{Y_{t}}$ is relatively ample over $A_{t}$ (this is a product situation). By taking relative extremal contractions in the sense of Mori theory one gets a tower of three $\mathbb{P}_{1}$-bundles. Of course there are three choices of the first one and then two choices for the second since the situation is completely symmetric in $i$. (This situation could possibly lead to some monodromy action $\pi_{1}\left(A_{t}\right) \rightarrow \mathfrak{S}_{3}$, but since such actions are discrete and depend continuously on $t$, the fact that we have a non twisted product for $t=0$ implies that we have no such twist for $t$ arbitrary). The last contraction will provide the space $y_{i}$ for the appropriate $i$. Now consider the canonical map

$$
Y_{t} \rightarrow Y_{1, t} \times_{A_{t}} Y_{2, t} \times_{A_{t}} Y_{3, t} .
$$

Then this map is immediately seen to be an isomorphism.

Since $Y_{i, t}$ is a $\mathbb{P}_{1}$-bundle over $A_{t}$ and since it is has a section by construction, it follows

$$
Y_{i, t}=\mathbb{P}\left(E_{i, t}\right)
$$

with a rank 2-bundle $E_{i, t}$ (normalized such that $E_{0, t}=\mathcal{O}_{A_{0}} \oplus L_{i}$ ), and the $E_{i, t}$ form a holomorphic rank 2-bundle $\mathcal{E}_{i}$ over $\mathcal{A}$. Since the section at infinity in $Y_{0}$ deforms by construction to sections in $Y_{t}$, one obtains a global quotient $\varepsilon_{i} \rightarrow g_{i} \rightarrow 0$ such that $g_{i} \mid A_{0}=\mathcal{O}_{A_{0}}$. By changing $\varepsilon_{i}$ appropriately, one may assume that $g_{i}=\mathcal{O}_{\mathcal{A}}$. Let $\mathcal{L}_{i}$ be the kernel of $\mathcal{E}_{i} \rightarrow \mathcal{O}_{\mathcal{A}}$. Then $\mathcal{L}_{i} \mid A_{0}=L_{i}$. But this implies that there is a deformation of $A=A_{0}$ such that all three line bundles $L_{i}$ remain holomorphic. But the assumption

$$
V_{1} \cap V_{2} \cap V_{3}=\{A\}
$$

implies that there is no such (nontrivial) deformation of $A$.

It is therefore a very natural question to ask whether these rigid 6-dimensional Kähler manifolds are projective or not. If they were not projective, we would get counter-examples to the Kodaira conjecture. Unfortunately (in view of getting easy counter-examples!), Theorem 4 of the next section tells us that a complex torus $A$ verifying Assumption $(*)$ for some triple of holomorphic line bundles $L_{i}$ is always an abelian variety. In fact, Theorem 4 even shows that $\left(\mathbb{P}_{1}\right)^{3}$-bundles of the special type

$$
Y=\mathbb{P}\left(\mathcal{O}_{A} \oplus L_{1}\right) \times_{A} \mathbb{P}\left(\mathcal{O}_{A} \oplus L_{2}\right) \times_{A} \mathbb{P}\left(\mathcal{O}_{A} \oplus L_{3}\right)
$$

satisfy the Kodaira conjecture, even without assumption $(*)$ for $L_{1}, L_{2}, L_{3}$ (see Lemma 6). 


\section{Holomorphic line bundles on complex tori}

Let $X$ be a complex torus of dimension $g$. As explained in [BL99], [LB92] $X$ admits a period matrix of the form $\left(\tau, \mathbf{1}_{g}\right)$ with $\tau \in M_{g}(\mathbb{C})$, the $g \times g$-matrices with entries in $\mathbb{C}$ such that $\operatorname{det}(\operatorname{Im} \tau) \neq 0$. Conversely every such matrix is the period matrix of a complex torus.

If $\Lambda \in \mathbb{C}^{g}:=V$ denotes the lattice generated by the columns of $\left(\tau, \mathbf{1}_{g}\right)$ the Néron-Severi group of $X$ may be described as

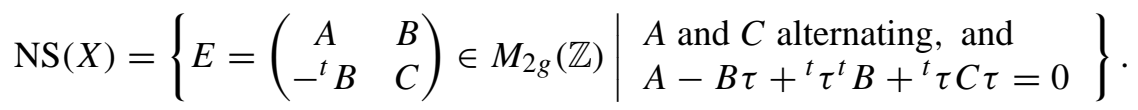

The equality ensures that the alternating form $E$ is a $(1,1)$-form, cf. [BL99, p. 10].

Theorem 4. Let $X$ be a 3-dimensional complex torus with period matrix $\left(\tau, \mathbf{1}_{3}\right)$ and let $E_{1} \cdot \mathbb{Z} \oplus E_{2} \cdot \mathbb{Z} \oplus E_{3} \cdot \mathbb{Z} \subset \mathrm{NS}(X)$ be a rank 3 subgroup of the Néron-Severi group $\mathrm{NS}(X)$ of $X$ generated by $E_{1}, E_{2}, E_{3} \in \mathrm{NS}(X)$. Then there is a sequence $\left(X_{n}\right)$ of 3-dimensional complex tori with period matrices $\left(\tau_{n}, \mathbf{1}_{3}\right)$ such that

(i) the $\tau_{n}$ converge to $\tau$ for $n \rightarrow \infty$,

(ii) $E_{1} \cdot \mathbb{Z} \oplus E_{2} \cdot \mathbb{Z} \oplus E_{3} \cdot \mathbb{Z} \subset \mathrm{NS}\left(X_{n}\right)$, and

(iii) $X_{n}$ is a complex abelian variety.

As a first step towards a proof, $E=\left(\begin{array}{cc}A & B \\ -{ }^{t} B & C\end{array}\right)$ may be considered as an element of the free abelian group $\mathbb{Z}^{15}$ : the matrices $A=\left(\begin{array}{ccc}0 & a_{1} & a_{2} \\ -a_{1} & 0 & a_{3} \\ -a_{2} & -a_{3} & 0\end{array}\right)$ and $C=$ $\left(\begin{array}{ccc}0 & c_{1} & c_{2} \\ -c_{1} & 0 & c_{3} \\ -c_{2} & -c_{3} & 0\end{array}\right)$ are alternating, and $B=\left(\begin{array}{lll}b_{1} & b_{2} & b_{3} \\ b_{4} & b_{5} & b_{6} \\ b_{7} & b_{8} & b_{9}\end{array}\right)$ is arbitrary. Since $k \cdot E \in \mathrm{NS}(X)$ implies $E \in \mathrm{NS}(X)$, condition (ii) is equivalent to

$$
E_{1} \cdot \mathbb{Q} \oplus E_{2} \cdot \mathbb{Q} \oplus E_{3} \cdot \mathbb{Q} \subset \mathrm{NS}\left(X_{n}\right) \otimes_{\mathbb{Z}} \mathbb{Q},
$$

and $E_{1} \cdot \mathbb{Q} \oplus E_{2} \cdot \mathbb{Q} \oplus E_{3} \cdot \mathbb{Q}$ may be interpreted as a $\mathbb{Q}$-rational point in the Grassmannian $G(3,15)$.

For a given 3-dimensional subspace $E_{1} \cdot \mathbb{Q} \oplus E_{2} \cdot \mathbb{Q} \oplus E_{3} \cdot \mathbb{Q} \subset \mathbb{Q}^{15}$ the equations $A_{i}-B_{i} \tau+{ }^{t} \tau^{t} B_{i}+{ }^{t} \tau C_{i} \tau=0, i=1,2,3$ imply algebraic relations between the entries of

$$
\tau=\left(\begin{array}{ccc}
\tau_{1} & \tau_{2} & \tau_{3} \\
\tau_{4} & \tau_{5} & \tau_{6} \\
\tau_{7} & \tau_{8} & \tau_{9}
\end{array}\right)
$$


Since the $A_{i}-B_{i} \tau+{ }^{t} \tau^{t} B_{i}+{ }^{t} \tau C_{i} \tau$ are alternating matrices, the number of these relations can be reduced to $9(i=1,2,3)$ :

$$
\begin{aligned}
& 0=a_{i 1}-b_{i 1} \tau_{2}-b_{i 2} \tau_{5}-b_{i 3} \tau_{8}+b_{i 4} \tau_{1}+b_{i 5} \tau_{4}+b_{i 6} \tau_{7} \\
& +c_{i 1}\left(\tau_{1} \tau_{5}-\tau_{2} \tau_{4}\right)+c_{i 2}\left(\tau_{1} \tau_{8}-\tau_{2} \tau_{7}\right)+c_{i 3}\left(\tau_{4} \tau_{8}-\tau_{5} \tau_{7}\right) \\
& 0=a_{i 2}-b_{i 1} \tau_{3}-b_{i 2} \tau_{6}-b_{i 3} \tau_{9}+b_{i 7} \tau_{1}+b_{i 8} \tau_{4}+b_{i 9} \tau_{7} \\
& +c_{i 1}\left(\tau_{1} \tau_{6}-\tau_{3} \tau_{4}\right)+c_{i 2}\left(\tau_{1} \tau_{9}-\tau_{3} \tau_{7}\right)+c_{i 3}\left(\tau_{4} \tau_{9}-\tau_{6} \tau_{7}\right) \\
& 0=a_{i 3}-b_{i 4} \tau_{3}-b_{i 5} \tau_{6}-b_{i 6} \tau_{9}+b_{i 7} \tau_{2}+b_{i 8} \tau_{5}+b_{i 9} \tau_{8} \\
& +c_{i 1}\left(\tau_{2} \tau_{6}-\tau_{3} \tau_{5}\right)+c_{i 2}\left(\tau_{2} \tau_{9}-\tau_{3} \tau_{8}\right)+c_{i 3}\left(\tau_{5} \tau_{9}-\tau_{6} \tau_{8}\right) .
\end{aligned}
$$

So there is an algebraic subset $U_{E_{1}, E_{2}, E_{3}}$ of $\mathbb{C}^{9}$ such that

$$
U_{E_{1}, E_{2}, E_{3}} \cap\left\{\tau \in \mathbb{C}^{9}: \operatorname{det}(\operatorname{Im} \tau) \neq 0\right\}
$$

describes all $\tau$ 's with $E_{1} \cdot \mathbb{Q} \oplus E_{2} \cdot \mathbb{Q} \oplus E_{3} \cdot \mathbb{Q} \subset \mathrm{NS}\left(X_{\tau}\right) \otimes_{\mathbb{Z}} \mathbb{Q}$ where $X_{\tau}$ is the complex torus corresponding to the period matrix $\left(\tau, \mathbf{1}_{3}\right)$. In particular, the union of all these $U_{E_{1}, E_{2}, E_{3}}$ is an algebraic family $U \subset G(3,15) \times \mathbb{C}^{9}$. Let $\bar{U} \subset G(3,15) \times \mathbb{P}^{9}$ denote the projective closure of $U$.

The heart of the proof is now a careful analysis of this family $\bar{U}$, especially of the fibers over $\mathbb{Q}$-rational points of $G(3,15)$. If they always contain an (analytically) dense subset of $\tau$ 's such that $X_{\tau}$ is a complex abelian variety, the theorem will follow.

The first observation is that all coefficients in the equations of $(*)$ are rational. Hence, $\mathbb{Q}$ is the field of definition of $\bar{U}$, i.e. there exists a $\mathbb{Q}$-scheme $\bar{U}_{\mathbb{Q}}$ such that $\bar{U}=\bar{U}_{\mathbb{Q}} \times \mathbb{Q}$ Spec $\mathbb{C}$. In particular, every fiber of $\bar{U}$ over a $\mathbb{Q}$-rational point of $G(3,15)$ has $\mathbb{Q}$ as field of definition, too.

Next, one computes a fiber $\bar{U}_{E_{1}, E_{2}, E_{3}}$ of $\bar{U}$ with sufficiently general entries in the matrices $E_{1}, E_{2}, E_{3}$. This can be done with the computer algebra program Macaulay2 ([GS], [EGSS02]). Setting

$$
\begin{array}{rlrl}
A_{1}= & =\left(\begin{array}{ccc}
0 & 0 & 0 \\
0 & 0 & 2 \\
0 & -2 & 0
\end{array}\right), & B_{1}=\left(\begin{array}{lll}
1 & 1 & 0 \\
1 & 1 & 2 \\
1 & 1 & 2
\end{array}\right), & C_{1}=\left(\begin{array}{ccc}
0 & 1 & 0 \\
-1 & 0 & 0 \\
0 & 0 & 0
\end{array}\right), \\
A_{2}=\left(\begin{array}{ccc}
0 & 1 & 2 \\
-1 & 0 & 1 \\
-2 & -1 & 0
\end{array}\right), & B_{2}=\left(\begin{array}{lll}
0 & 0 & 0 \\
1 & 1 & 1 \\
0 & 1 & 0
\end{array}\right), & C_{2}=\left(\begin{array}{ccc}
0 & 0 & 0 \\
0 & 0 & 1 \\
0 & -1 & 0
\end{array}\right), \\
A_{3}=\left(\begin{array}{ccc}
0 & 1 & 2 \\
-1 & 0 & 1 \\
-2 & -1 & 0
\end{array}\right), & B_{3}=\left(\begin{array}{lll}
1 & 1 & 1 \\
1 & 2 & 1 \\
1 & 2 & 1
\end{array}\right), & C_{3}=\left(\begin{array}{ccc}
0 & 0 & 1 \\
0 & 0 & 0 \\
-1 & 0 & 0
\end{array}\right)
\end{array}
$$

(the matrix entries were chosen by a random number generator) and using the following Macaulay2 script 
Vol. 80 (2005)

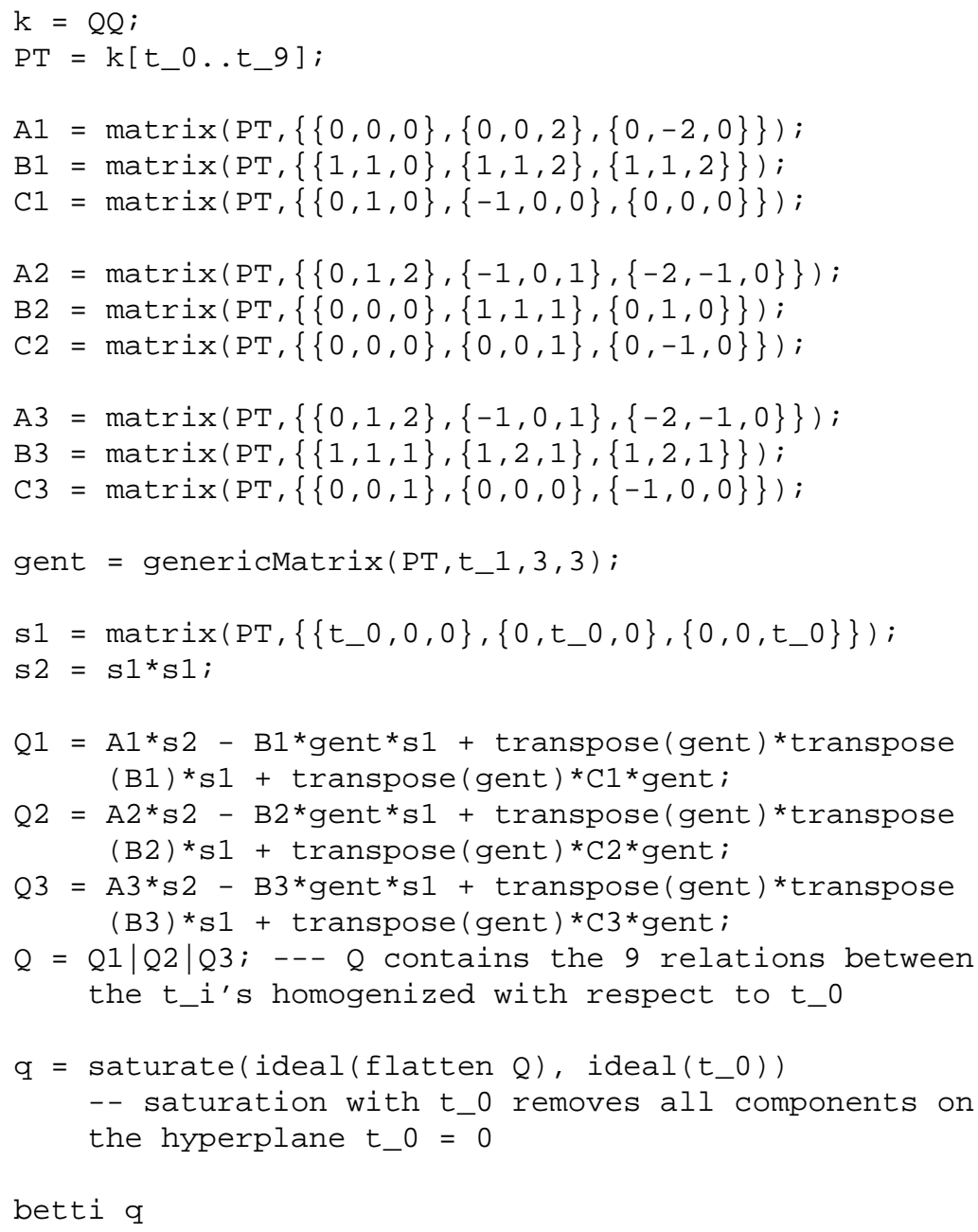

one gets 8 linear and 1 quadratic equation describing the projective closure of $U_{E_{1}, E_{2}, E_{3}}$ :

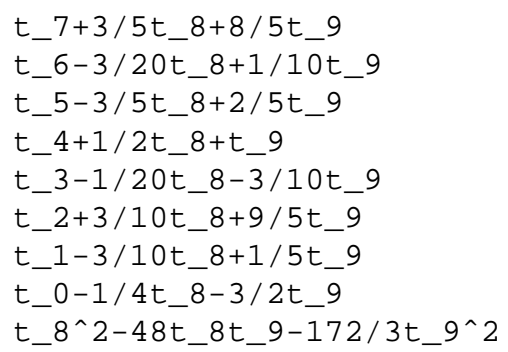

Since the quadratic generator has discriminant $24^{2}+4 \frac{172}{3}>0$ which is not the square 
of a rational number, this is a $\mathbb{Q}$-irreducible 0 -dimensional scheme of degree 2; over $\mathbb{C}$ it consists of two points.

Unfortunately, these equations may cut out too much, since the projective closure of a fiber may be less than the fiber of the projective closure of a family. To deal with this problem one has to do a little detour: First one looks at the (inhomogeneous) ideal of the whole family $U$ pulled back to $\left(\mathbb{A}^{12}\right)^{3} \times \mathbb{P}^{9}$ where each $\mathbb{A}^{12}$ parametrizes triples $A, B, C$ with $C$ normalized. This pull back is necessary since otherwise one has to embed $G(3,15)$ in some projective space which makes the computations impossible.

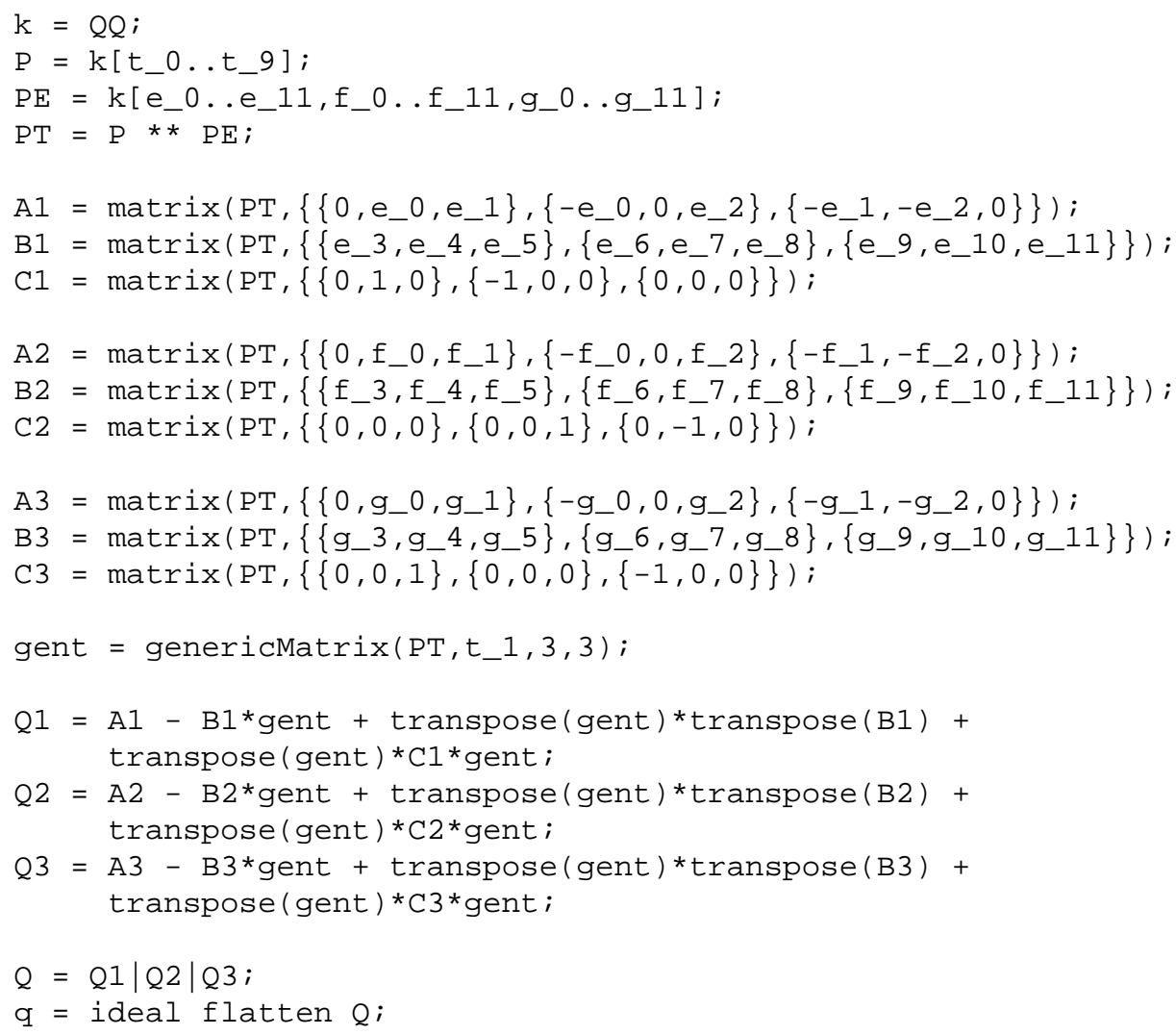

The projective closure of $U$ may be determined by computing a Groebner basis of this ideal with respect to a monomial order refining the order by degree in the $t_{i}$ 's and then homogenizing the generators with respect to $t_{0}$ ([Eis95, 15.31]). This computation is too complicated for the whole Groebner basis, but it is already enough to look at the first few elements which are added to the original generators:

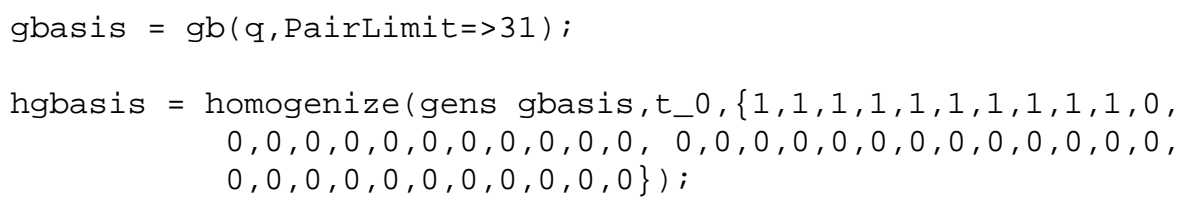




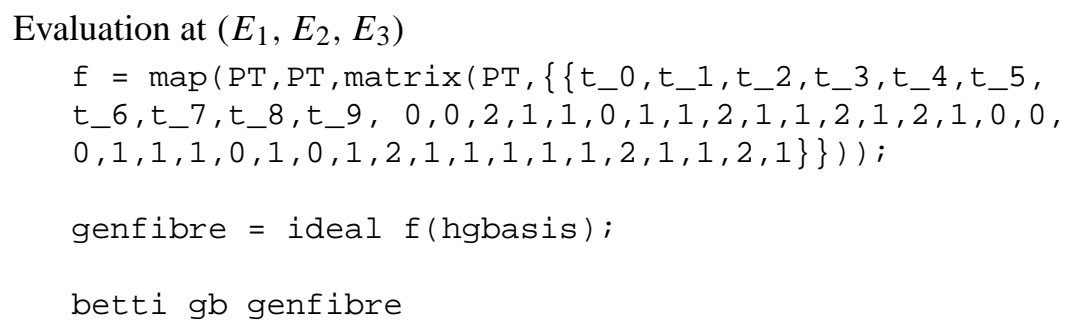

shows that the fibre $(\bar{U})_{E_{1}, E_{2}, E_{3}}$ is contained in a scheme cut out by 8 linear and 1 quadratic equation, so

$$
(\bar{U})_{E_{1}, E_{2}, E_{3}}=U_{E_{1}, E_{2}, E_{3}} \text {. }
$$

One can get further information about $\bar{U}$ from the homogenized equations collected in hgbasis. Since the projective closure of a fiber is equal to the fiber of the projective closure on an open subset they contain 9 equations describing the fibers of $\bar{U}$ over an open subset around $\left(E_{1}, E_{2}, E_{3}\right)$. Furthermore the command

transpose leadTerm hgbasis

shows that all of these equations contain $t$-variables. Hence each of these fibers is cut out by 9 non-constant equations, so it is not empty. Consequently, no fiber is empty. Turning to the fibers of $\bar{U}$ over $\mathbb{C}^{9}$ (resp. $\mathbb{P}^{9}$ ) one sees immediately that these are nonempty linear subspaces. Hence $\bar{U}$ is connected. Finally, the regularity of $U$ follows directly by deriving the equations in $(*)$ with respect to the $a_{i j}$ 's. Taken all these facts together it follows that $U$ and hence $\bar{U}$ is irreducible. So every 0 -dimensional fiber must have degree 2 .

Now it is easy to prove for these 0 -dimensional fibers over $\mathbb{Q}$-rational points that they describe period matrices $\tau$ belonging to complex abelian varieties: Since the fibers are $\mathbb{Q}$-rational, too, the entries of $\tau$ are elements of a field extension of $\mathbb{Q}$ of degree 2. The defining equations of the Néron-Severi group show that then $\operatorname{NS}\left(X_{\tau}\right)$ is a $15-2 \times 3=9$-dimensional $\mathbb{Q}$-vector space. But a 3 -dimensional complex torus with maximal Picard number 9 is algebraic (cf. [BL99]).

What about the higher dimensional fibers? We consider the $\mathbb{Q}$-rational map $\phi: G(3,15) \rightarrow \operatorname{Hilb}^{2}\left(\mathbb{P}_{\mathbb{Q}}^{9}\right)$ whose existence is the essence of the arguments used above. Let

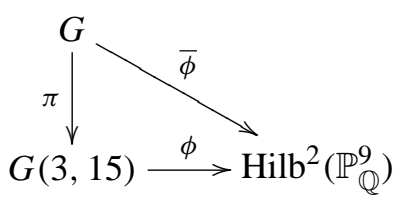

be the resolution of the singularities of $\phi$ by blowing up centers smooth over $\mathbb{Q}$. This is possible by the Hironaka package, see [Hir64], [BM97] or [HLOQ97]. Now the theorem is a consequence of the following result. 
Lemma 5. Let $Z \subset Y$ be an embedding of regular $\mathbb{Q}$-schemes, let $z \in Z$ be a $\mathbb{Q}$-rational point and let $\phi: \tilde{Y} \rightarrow Y$ be the blow up of $Y$ with center $Z$. Then the $\mathbb{Q}$-rational points are dense on the fiber $\phi^{-1}(z)$.

Proof. This is almost trivial: Choose a regular sequence $\left(f_{1}, \ldots, f_{s}, f_{s+1}, \ldots, f_{t}\right)$ in the local ring $\mathcal{O}_{Y, z}$ defined over $\mathbb{Q}$ such that $\mathfrak{m}_{Z, z}=\left(\bar{f}_{s+1}, \ldots, \bar{f}_{t}\right) \subset \mathcal{O}_{Z, z}$ and $\mathfrak{m}_{Y, Z}=\left(f_{1}, \ldots, f_{t}\right)$. The blowing up of $\operatorname{Spec} \mathcal{O}_{Y, Z}$ with center $\operatorname{Spec} \mathcal{O}_{Z, Z}$ is given by

$$
\operatorname{Proj} \mathcal{O}_{Y, z}\left[f_{s+1}, \ldots, f_{t}\right]=\left(\operatorname{Spec} \mathcal{O}_{Y, z} \times \mathbb{P}_{\mathbb{Q}}^{t-s-1}\right) / V\left(T_{i} f_{j}-T_{j} f_{i}\right),
$$

and the fiber over $z$ is $\cong \mathbb{P}_{\mathbb{Q}}^{t-s-1}$.

Apply the lemma on $\phi$ : If $\left[E_{1} \cdot \mathbb{Q} \oplus E_{2} \cdot \mathbb{Q} \oplus E_{3} \cdot \mathbb{Q}\right]=[W] \in G(3,15)$ is a $\mathbb{Q}$-rational point then $\pi^{-1}([W]) \subset G$ will contain an analytically dense subset of $\mathbb{Q}$-rational points, and the same will be true of the image $\bar{\phi}\left(\pi^{-1}([W])\right) \subset \operatorname{Hilb}^{2}\left(\mathbb{P}_{\mathbb{Q}}^{9}\right)$. But $\mathbb{Q}$-rational points in $\operatorname{Hilb}^{2}\left(\mathbb{P}_{\mathbb{Q}}^{9}\right)$ describe pairs of points corresponding to abelian varieties, and all pairs in $\bar{\phi}\left(\pi^{-1}([W])\right)$ map surjectively on the fiber over $[W]$ in $\bar{U}$. Hence this fiber contains a dense open subset of period matrices $\tau$ such that $X_{\tau}$ is an abelian variety.

Remark. Some words about the Macaulay2 computations: Since all the relevant equations and varieties are defined over $\mathbb{Q}$ and also the operations applied to them like taking the projective closure work over $\mathbb{Q}$, these calculations give exact results.

\section{Modifications and a general setting for counter-examples}

Of course the construction in Section 1 possibly could be modified in several ways and then might lead to a counter-example to the Kodaira conjecture.

First we show that even without Assumption (*) the variety $X$ constructed as before Proposition 3 is algebraically approximable. Indeed in that situation (using the previous terminology), $V_{1} \cap V_{2} \cap V_{3}$ contains other complex tori than $A$. Then theorem 4 assures the existence of a sequence $\left\{A_{n}\right\}_{n \in \mathbb{N}} \subset V_{1} \cap V_{2} \cap V_{3}$ of abelian varieties converging to $A$. The following lemma shows that this implies $X$ almost algebraic:

Lemma 6. Let $E=\left(\begin{array}{cc}A & B \\ -{ }^{t} B & C\end{array}\right) \in M_{2 g}(\mathbb{Z})$ be a skew symmetric matrix with integral entries and let

$$
V=\left\{\tau \in M_{3}(\mathbb{C}) \mid A-B \tau+{ }^{t} \tau^{t} B+{ }^{t} \tau C \tau=0 ; \operatorname{det} \operatorname{Im} \tau \neq 0\right\} \subset \mathbb{C}^{9}
$$

be the set of period matrices $\tau$ such that $X_{\tau}$ is a complex torus with $E \in \operatorname{NS}\left(X_{\tau}\right)$. 
Let $\mathcal{X}=V \times \mathbb{C}^{g} / \Lambda_{\tau}$ be the family of these tori $X_{\tau}$ where $\Lambda_{\tau}=\left(\tau, \mathbf{1}_{g}\right)$ is the lattice belonging to $X_{\tau}=\mathbb{C}^{g} / \Lambda_{\tau}$. Then every $\tau_{0} \in V$ has an open neighborhood $U \subset V$ such that there exists a holomorphic line bundle $\mathcal{L}_{U}$ on $\mathcal{X}_{U}$ such that $c_{1}\left(\mathcal{L}_{\tau}\right)=E$ for all $\tau \in U$.

Proof. Let $\pi: \mathcal{X} \rightarrow V$ be the projection of $\mathcal{X}$ onto $V$. By taking direct images with respect to $\pi$ and deriving the long exact sequence from $0 \rightarrow \mathbb{Z} \rightarrow \mathcal{O}_{X} \rightarrow \mathcal{O}_{X}^{*} \rightarrow 1$ one obtains the sequence

$$
R^{1} \pi_{*} \mathcal{O}_{X}^{*} \rightarrow R^{2} \pi_{*} \mathbb{Z} \rightarrow R^{2} \pi_{*} \mathcal{O}_{X}
$$

The skew symmetric matrix $E$ gives a section of $R^{2} \pi_{*} \mathbb{Z}$ which is mapped to 0 in $R^{2} \pi_{*} \mathcal{O}_{x}$ since $E \in \operatorname{NS}\left(X_{\tau}\right)$ for all $\tau \in V$. Hence $E$ is the image of a section in $R^{1} \pi_{*} \mathcal{O}_{X}^{*}$. Take an open neighborhood $U$ of $\tau_{0}$ such that the section restricted to $U$ is a cohomology class in $H^{1}\left(\mathcal{X}_{\mid \pi^{-1}(U)}, \mathcal{O}_{X}^{*}\right)$. This class gives the line bundle $\mathscr{L}_{U}$.

Next, consider the following more general setting: Take an $n$-dimensonal complex torus $A$ and $k$ vector bundles $E_{1}, \ldots, E_{k}$ over $A$ of rank $r_{1}, \ldots, r_{k} \leq n$. Let $Y$ be the $\left(n+r_{1}+\cdots+r_{k}\right)$-dimensional manifold

$$
\mathbb{P}\left(\mathcal{O}_{A} \oplus E_{1}\right) \times_{A} \cdots \times_{A} \mathbb{P}\left(\mathcal{O}_{A} \oplus E_{k}\right) .
$$

This a $\left(\mathbb{P}^{r_{1}} \times \cdots \times \mathbb{P}^{r_{k}}\right)$-bundle over $A$ with projection $\pi: Y \rightarrow A$. In each subspace $\mathbb{P}\left(\mathcal{O}_{A} \oplus E_{i}\right)$ there is a section $Z_{i}$ at infinity given by the direct summand $\mathcal{O}_{A}$. This gives a section $Z$ of $\pi$ by selecting over every $a \in A$ the point $\left(x_{1}, \ldots, x_{k}\right)$, where $\left\{x_{i}\right\}=Z_{i} \cap \pi^{-1}(a)$. Let $\sigma: X \rightarrow Y$ be the blow up of $Z \subset Y$.

Similar arguments as in Section 1 show

Proposition 7. If there is a positive-dimensional family of deformations of $X$ then there will also exist a deformation family of complex tori $\left\{A_{t}\right\}_{t \in \Delta}$ such that $A=A_{0}$ and all vector bundles $E_{1}, \ldots, E_{k}$ remain holomorphic on $A_{t}$.

The condition on the vector bundles to remain holomorphic requires some further explanations: Let $E$ be a vector bundle of rank $r$ over an $g$-dimensional torus $A$. Then the Chern classes $c_{i}(E)$ are $(i, i)$-classes in $H^{2 i}(A, \mathbb{Z})=\bigwedge^{2 i} \operatorname{Hom}(\Lambda, \mathbb{Z})$, where $\Lambda \subset \mathbb{C}^{g}=: V$ is a (non-degenerate) lattice such that $A=V / \Lambda$. Since $H^{i, i}(A, \mathbb{C})=\bigwedge^{i} \operatorname{Hom}_{\mathbb{C}}(V, \mathbb{C}) \times \bigwedge^{i} \operatorname{Hom}_{\overline{\mathbb{C}}}(V, \mathbb{C})$, the $(i, i)$-classes in $H^{2 i}(A, \mathbb{Z})$ may be interpreted as a real valued alternating form $F$ on $\bigwedge^{i} V$ such that

$$
F(i \Phi, i \Psi)=F(\Phi, \Psi) \quad \text { and } \quad F\left(\bigwedge^{i} \Lambda, \bigwedge^{i} \Lambda\right) \subset \mathbb{Z}
$$


As in the case of $(1,1)$-classes these conditions induce relations between $F$ (written in terms of a base of $\Lambda$ ) and the period matrix $\tau$. In a family $\left\{A_{t}\right\}_{t \in \Delta}$ of complex tori these relations must be satisfied for $\tau_{t}, t \neq 0$, if a holomorphic vector bundle $E$ on $A_{0}$ still has a holomorphic structure on $A_{t}$.

But the existence problem for vector bundles of higher rank with prescribed Chern classes is much more difficult than in the case of line bundles. On (non-algebraic) complex tori this problem is completely solved only in dimension 2 and rank 2 [Tom99], [TT02]. Consequently, to construct a counter-example to Kodaira's conjecture with vector bundles of higher rank it is not enough to give a set of Chern classes and to prove that these classes can be Chern classes only for isolated period matrix. On the other hand if there is a positive family of such period matrices there may be still a counter-example depending on the existence of vector bundles with these Chern classes only on isolated members of the families.

Finally the two simplest cases of this general setting are considered.

3.1. Line bundles in arbitrary dimensions. Let $X$ be a complex torus of dimension $g$ given by the period matrix $\left(\tau, \mathbf{1}_{g}\right)$. By the characterization of the Néron-Severi group in the last section a skew symmetric matrix $E \in M_{2 g}(\mathbb{Z})$ is a $(1,1)$-form iff the entries of $\tau$ satisfy $\left(\begin{array}{l}g \\ 2\end{array}\right)$ equations. Consequently, 3 skew symmetric matrices $E_{1}, E_{2}, E_{3} \in M_{2 g}(\mathbb{Z})$ should determine at most a finite number of $g \times g$ period matrices $\tau$ such that $E_{1}, E_{2}, E_{3}$ are first Chern classes of line bundles on $X_{\tau}$.

As in the last section, for given $g$ one can choose random entries for $E_{1}, E_{2}, E_{3}$ and compute the locus $V\left(E_{1}\right) \cap V\left(E_{2}\right) \cap V\left(E_{3}\right)$ of $\tau$ 's as above. But already in dimension 4 this locus turns out to be empty for randomly chosen entries. This means that only special triples of matrices belong to the Néron-Severi group of a complex torus, and it seems difficult to find one such that furthermore the above locus consists of isolated points. And then one has still to prove that the period matrices in this locus determine a non-algebraic complex torus.

3.2. Rank 2 vector bundles in dimension 3. This is the simplest case with vector bundles of rank $>1$. Unfortunately, by Poincaré duality

$$
H^{2,2}(X, \mathbb{Z}) \cong H^{1,1}(X, \mathbb{Z}), H^{3,1}(X, \mathbb{Z}) \cong H^{0,2}(X, \mathbb{Z}), H^{1,3}(X, \mathbb{Z}) \cong H^{2,0}(X, \mathbb{Z})
$$

and the equations for a skew symmetric matrix in $M_{2 g}(\mathbb{Z})$ to be a $(2,2)$-form do not differ from those for $(1,1)$-forms. Hence in this case a counter-example may be found only by closer considering the question for which complex tori exist rank 2 vector bundles with given Chern classes.

Of course more difficult settings starting with rank 2 vector bundles on 4-dimensional complex tori may give positive results. On the other hand the examples above 
give enough evidence to turn around one's point of view, into an attempt to prove Kodaira's conjecture in these special cases.

\section{Deformations of Projective Bundles}

In this final section we generalize Proposition 2.

Theorem 8. Let $X$ be a compact manifold which has a $\mathbb{P}_{r}$-bundle structure $X \rightarrow Y$ over some compact manifold $Y$. Then for every deformation $X \rightarrow S$ with $X_{0} \simeq X$, the nearby fibers $\mathcal{X}_{t}$ have a $\mathbb{P}_{r}$-bundle structure $\mathcal{X}_{t} \rightarrow \mathcal{Y}_{t}$ where $\mathcal{Y}_{\mathrm{i}}$ is a deformation of $Y$ in a neighborhood of $t=0$. Moreover, if $X=\mathbb{P}(V)$ for some vector bundle $V$ on $Y$, then $\mathcal{X}_{t}=\mathbb{P}\left(V_{t}\right)$ for a suitable deformation $V_{t} \rightarrow y_{t}$ of $V \rightarrow Y$.

Proof. Let $q: \mathcal{C} \rightarrow \mathcal{T}$ be the irreducible component of the cycle space relative to $\pi: \mathcal{X} \rightarrow S$ containing the fibers of $X \rightarrow Y$. So $\mathcal{T}$ parametrizes deformations of the $\mathbb{P}_{r}$ to nearby fibers $X_{y}$. Since the normal bundle in $\mathcal{C}$ to these projective spaces is trivial, it follows immediately that (possibly after shrinking $S$ ) $\mathcal{T}$ is smooth. Let $p: \mathcal{C} \rightarrow \mathcal{X}$ denote the projection and notice that there is another canonical projection $r: \mathcal{T} \rightarrow S$ realizing $\mathcal{T}$ as a family $\left(T_{s}\right)$. We will also consider $\mathcal{C}_{s}=q^{-1}\left(T_{s}\right)$ with projection $q_{s}$ to $T_{s}$. Now $q_{0}$ is a $\mathbb{P}_{r}$-bundle. Therefore for small $s$ also the maps $q_{s}$ are first submersions and second projective bundles (since projective space is locally rigid). Having in mind that $p_{0}: \mathcal{C}_{0} \rightarrow X_{0}$ is an isomorphism, we see that $p$ is an isomorphism so that all $X_{t}$ are projective bundles for small $t$.

The vector bundle statement finally is proved just as in Proposition 2.

\section{References}

[BM97] E. Bierstone and P. D. Milman, Canonical desingularization in characteristic zero by blowing up the maximum strata of a local invariant. Invent. Math 128 (1997), 207-302. Zbl 0896.14006 MR 1440306

[BL99] Ch. Birkenhake and H. Lange, Complex Tori. Progr. Math. 177. Birkhäuser, Boston 1999. Zbl 0945.14027 MR 1713785

[DPS96] Jean-Pierre Demailly, Thomas Peternell and Michael Schneider, Compact Kähler manifolds with Hermitian semipositive anticanonical bundle. Compositio Math. 101 (1996), 217-224. Zbl 1008.32008 MR 1389367

[Eis95] D. Eisenbud, Commutative Algebra with a View Toward Algebraic Geometry. Grad. Texts in Math. 150, Springer-Verlag, Berlin 1995. Zbl 0819.13001 MR 1322960

[EGSS02] D. Eisenbud, D. Grayson, M. Stillman, and B. Sturmfels (eds.), Computations in Algebraic Geometry with Macaulay 2. Algorithms Comput. Math. 8, SpringerVerlag, Berlin 2002. Zbl 0973.00017 MR 1949544 
[GS] D. Grayson and M. Stillman, Macaulay 2. A software system for research in algebraic geometry and commutative algebra. Available in source code form and compiled for various architectures, with documentation, at http: / / www . math . uiuc. edu/Macaulay2/.

[HLOQ97] H. Hauser, J. Lipman, F. Oort, and A. Quirós (eds.), Resolution of Singularities. Progr. Math. 181, Birkhäuser, Basel 1997. Zbl 0932.00042 MR 1748614

[Hir64] H. Hironaka, Resolution of singularities of an algebraic variety over a field of characteristic zero I, II. Ann. Math. 79 (1964), 109-203; ibid. 205-326. Zbl 0122.38603 MR 0199184

[Kod63] K. Kodaira, On compact analytic surfaces III. Ann. Math. 78 (1963), 1-40. Zbl 0171.19601 MR 0184257

[LB92] H. Lange and Ch. Birkenhake, Complex Abelian Varieties. Grundlehren Math. Wiss. 302, Springer-Verlag, Berlin 1992. Zbl 0779.14012 MR 1217487

[Og04] K. Oguiso, Groups of automorphisms of null-entropy of hyperkähler manifolds. math.AG/0407476

[TT02] A. Teleman and M. Toma, Holomorphic vector bundles on non-algebraic surfaces. C. R. Math. Acad. Sci. Paris 334 (2002), 383-388. MR 1892939

[Tom99] M. Toma, Stable bundles with small $c_{2}$ over 2-dimensional complex tori. Math.Z. 232 (1999), 511-525. Zbl 0945.32007 MR 1719686

[Vo04a] C. Voisin, On the homotopy types of compact Kähler and complex projective manifolds. Invent. Math. 157 (2004), 329-343. MR 2076925

[Vo04b] C. Voisin, On the homotopy types of Kähler manifolds and the birational Kodaira problem. math.AG/0410040

Received March 10, 2003

Jean-Pierre Demailly, Institut Fourier, Université de Grenoble I, BP 74, 38402 St. Martin d'Hères, France

E-mail: Jean-Pierre.Demailly@ujf-grenoble.fr

Thomas Eckl, Mathematisches Institut, Universität zu Köln, Weyertal 86-90, 50931 Köln, Germany

E-mail: thomas.eckl@math.uni-koeln.de

Thomas Peternell, Institut für Mathematik, Universität Bayreuth, 95440 Bayreuth, Germany

E-mail: thomas.peternell@uni-bayreuth.de 